\title{
Robustness Analysis of Classical High Voltage Joint Design Under High Voltage DC Stress
}

\author{
Fredrik Fälth \\ ABB High Voltage Cables \\ Karlskrona, Sweden \\ fredrik.falth@se.abb.com
}

\author{
Santhosh Kumar BVMP \\ $A B B$ GISL \\ Chennai, India \\ santhosh.bvmp@in.abb.com
}

\author{
Hossein Ghorbani \\ ABB High Voltage Cables \\ Karlskrona, Sweden \\ hossein.ghorbani@se.abb.com
}

\begin{abstract}
The demand for extruded HVDC cable systems for higher voltages has been increasing in different fields. This demand comes from the need for higher transmission capacity in applications such as integration of wind power, power system interconnectors and connection of offshore oil and gas platforms to land. Prefabricated joints are used to connect the cable delivery lengths. If badly designed, the possibility of breakdown in the joints is higher than in other parts of the system because of the number of interfaces and triple points between different materials. Due to the huge potential costs and the down-time imposed to the system in case of a joint failure, the robustness of the joints is of great importance. Two main approaches in the design of prefabricated joints for extruded cables exist in which the more recent one takes advantage of the nonlinear properties of modern field grading materials. In this work, we have examined the design challenges with focus on robustness issues in a $150 \mathrm{kV}$ classical joint design without field grading material. The results show that with this approach, there are serious robustness issues which are challenging to overcome without field grading material.
\end{abstract}

\section{Introduction}

The market for extruded High Voltage DC (HVDC) cable connections has increased dramatically during the last decade, driven mainly by interconnectors, wind farms and power supply to oil-and-gas fields [1]. In the pursuit for higher transmission capacity, the system voltage has steadily been raised from $80 \mathrm{kV}$ [2], to 320 $\mathrm{kV}$ HVDC systems currently being installed [3]. The increased voltage level greatly enhances the demand on cable system performance and reliability. Besides the cable, the quality of the accessories such as terminations and prefabricated joints, play an important role. In particular, special care should be taken to the design of the HVDC joints. Due to the number of joints in HVDC systems, the design must be cost effective on one hand, while on the other hand, a joint breakdown is very costly due to high repair costs and the long downtimes involved. Moreover, the cost of a joint breakdown is considerably increased if it occurs at sea. For these reasons, the robustness of the joints is of utmost importance. To be able to withstand the increased electric stress, careful design of the accessories becomes more and more important. The electric field must be controlled in such a way that it does not exceed the tolerance level of the respective material in any part of the system. In AC systems, the main methods for field control are geometric and capacitive field grading [4]. In DC applications, resistive field grading is common, where the field is re-distributed by controlling the leakage current flow. In practice, this approach can be complicated since the resistivity of rubber materials is strongly dependent on temperature and electric field [5]. Initially, when HVDC was introduced, the experiences and materials developed for HVAC were also employed for DC accessories. Later, gradually the same materials have been optimized for DC applications. Lately, a new class of resistive field grading materials (FGM) with nonlinear resistivity has been introduced [4] and [6]. The nonlinear behavior of this material gives the possibility to create more robust designs [7]. However, cable accessory producers still rely on the classical AC designs, without nonlinear field grading materials, adopted for DC applications.

Within this work a classical AC joint design, adapted for DC, is simulated in order to study design challenges with this approach. The behavior of the joint, with realistic material parameters, under different stresses was investigated. The joint was tuned to the case with the highest thermal and electrical stresses, and then simulated under different thermal and electrical stress conditions in order to examine its robustness. As will be described below, the main issue with this design approach is the interface field between the joint and cable insulation. The electric field at this interface is governed by the surrounding material properties which are very sensitive to changes in temperature and electric field which may cause serious robustness issues.

\section{Model}

\subsection{Geometry}

All the simulations are done using COMSOL Multiphysics 4.2 with 2D "Axisymmetric" geometry, see Fig. 1. This is a generic joint geometry where insulating material is introduced between the conducting inner and outer deflectors, effectively separating high voltage from ground. The deflector shape is designed considering the impulse test requirements. The cable consists of a $1500 \mathrm{~mm}^{2}$ conductor with $12 \mathrm{~mm}$ 
insulation layer thickness. The length of the full joint is $944 \mathrm{~mm}$, with an outer radius of $192 \mathrm{~mm}$.

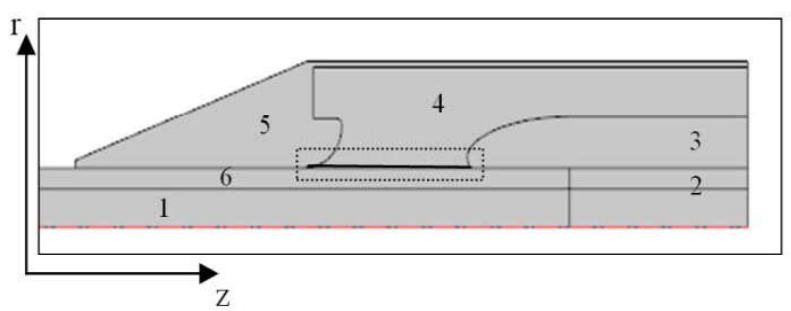

Fig. 1. Generic joint design used in the simulations; 1conductor, 2-connector, 3-inner deflector, 4-joint insulation, 5-outer deflector, 6-cable insulation. The dotted box indicates the interface between joint and cable insulation materials.

\subsection{Materials}

The cable has an aluminum conductor and XLPE insulation. The conductivity is a function of temperature and electric field as shown below

$$
\sigma(E, T)=\sigma_{0} \exp \left(\alpha\left(T-T_{\text {Ref }}\right)+\beta|E|\right) \quad \text { Eq. } 1
$$

with parameters assumed in the range of $\sigma_{0}=10^{-16} \mathrm{~S} / \mathrm{m}$, $\alpha=0.1 \mathrm{~K}^{-1}, T_{\mathrm{Ref}}=293 \mathrm{~K}$, and $\beta=10^{-7} \mathrm{~m} / \mathrm{V}[8]$. The joint insulation is a high voltage grade EPDM where the conductivity level is also described by Eq. 1, with parameters assumed in the range of $\sigma_{0}=5 \cdot 10^{-15} \mathrm{~S} / \mathrm{m}, \alpha$ $=0.02 \mathrm{~K}^{-1}, T_{\mathrm{Ref}}=293 \mathrm{~K}, \beta=5 \cdot 10^{-8} \mathrm{~m} / \mathrm{V}$ [8]. Finally, joint deflectors are made of semi-conducting EPDM material.

\subsection{Physics}

The problem must be simulated with coupled thermal and electrical equations. Both dynamic and static simulations are required to predict the transient and steady state behavior of the design.

Maxwell equations in case of DC can be simplified and rewritten as below:

$$
-\nabla \cdot \frac{\partial\left(\varepsilon_{0} \varepsilon_{\mathrm{r}} \nabla V\right)}{\partial t}-\nabla \cdot(\sigma \nabla V)=0
$$

In which, $\varepsilon_{\mathrm{r}}$ is the relative permittivity and $\sigma$ is the conductivity which is assumed to be a function of electric field and temperature i.e., $\sigma(T, E)$.

Thermal dynamics are calculated by the heat transfer equation:

$$
\rho_{\mathrm{M}} c_{\mathrm{p}} \frac{\partial T}{\partial t}+\nabla \cdot(-\lambda \nabla T)=Q
$$

In which, $\lambda$ is thermal conductivity, $\rho_{\mathrm{M}}$ is the density specific heat, and $c_{\mathrm{p}}$ is the specific heat. $Q$ is the heat source. For the cable conductor heating, it is calculated as:

$$
Q=\frac{I_{\mathrm{c}}^{2} \rho_{\mathrm{c}}\left(1+\alpha_{\mathrm{Th}}\left(T-T_{0}\right)\right)}{A}
$$

and resistive heating in the insulation media is:

$$
Q=j E
$$

Eq. 5
It is remarked that Eq. 5 leads to a strong coupling of Eq. 2 and Eq. 3. As for the electrical boundary conditions, Dirichlet boundary condition is applied at the conductor interface (applied voltage) and ground; a zero flux boundary condition is applied at the symmetry line and the boundary between the insulations.

For thermal boundary conditions, we used realistic thermal resistance from the joint body to ambient temperature to mimic the convection.

\subsection{Simulations}

A cable system has to undergo various tests before it is qualified. According to CIGRE WG 21-01 [9], an extruded cable system has to pass a Type Test (TT) and a Long Term Test (LTT) in order to be qualified. The TT includes a load cycling test with voltage, $U_{\mathrm{T}}$, equal to $1.85 \cdot U_{0}$, where $U_{0}$ is the rated $\mathrm{DC}$ voltage. The voltage in the LTT, $U_{\mathrm{T} 1}$, is $1.45 \cdot U_{0}$.

The TT also includes a series of switching impulse (SI) tests and it can include lightning impulse (LI) tests depending on the application. The impulse voltage requirements for a $150 \mathrm{kV}$ cable system are visualized in Fig. 2. The SI waveform has a standard time to crest of $t_{\mathrm{cr}}=250 \mu \mathrm{s} \pm 20 \%$, and time to half value in the tail is $t_{2}=2500 \mu \mathrm{s} \pm 60 \%$. All the tests are conducted under a nominal current load.

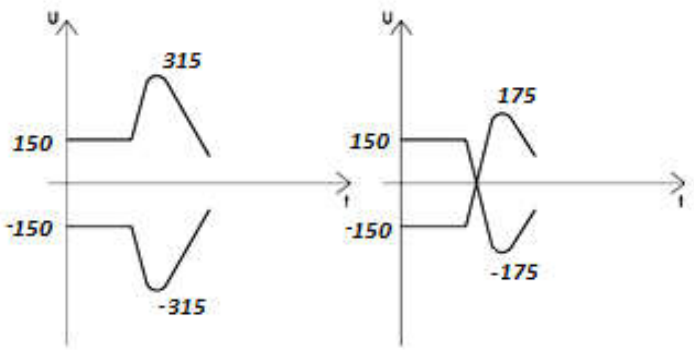

Fig. 2. Switching impulse voltages for a $150 \mathrm{kV}$ HVDC cable system.

Finite element method (FEM) analysis on breakdown phenomenon of cable joint with impurities, are reported in [10] and [11]. Within this work, using FEM, we have simulated the electrical TT, which gives the most critical conditions. The joint behavior at TT voltage $\left(\mathrm{U}_{\mathrm{T}}\right)$ is studied at steady state for both high and low operational temperatures. Furthermore, the response of the joint to the superimposed DC and Switching Impulse with Same Polarity (SISP) and Opposite Polarity (SIOP) is simulated in order to capture the transient behavior of the system. Finally, the robustness of the joint with respect to cable insulation resistivity, as well as thermal boundary conditions, has been examined.

\section{Results}

Generally, the most critical regions in high voltage devices are triple points, as well as the interfaces between different materials. At these locations different material properties lead to space charge accumulation 
which in combination with edges enhances the electric field. In HVDC joints, the interface between the cable and joint insulations is the most critical region; especially without sufficient interface pressure, the interface will have low breakdown strength against high tangential fields [12]. Generally, the interfacial pressure is known to be a major factor determining the breakdown strength. It is further complicated as the interfacial pressure may be reduced during the thermal cycling [13]. Furthermore, other factors such as lubricating grease at the interface and the length of the interface also affect the possibility of breakdown [12].

Due to the facts above, this study was mainly focused on the tangential electric field at the insulation interface (illustrated in the dotted box in Fig. 1).

\subsection{DC simulations}

Electric field calculations at DC in steady state are performed for cold and hot conditions. In cold condition, the whole system is kept at $20{ }^{\circ} \mathrm{C}$. In the hot condition, the cable conductor is heated to $70{ }^{\circ} \mathrm{C}$, and temperature of the other parts is governed via the heat transfer equations. Fig. 3 shows a surface plot of the temperature distribution in the joint in the hot condition.

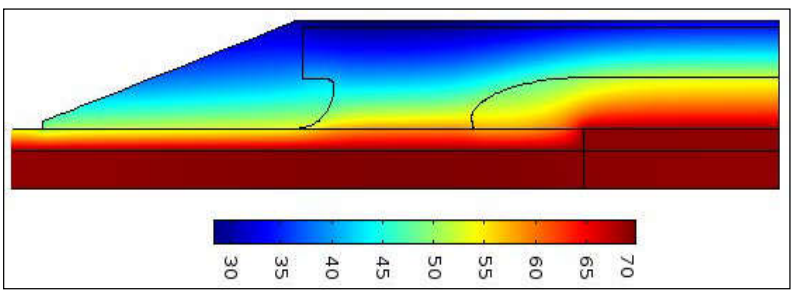

Fig. 3. Temperature distribution in the joint under DC voltage and conductor heating.

As a starting point, the material properties were set according to typical XLPE and EPDM conductivity levels and the electric fields at cold and hot conditions during the TT were calculated. The resulting voltage distributions are shown in Fig. 4. At room temperature, the field in the cable and joint insulation is higher close to the conductor. In contrast, at hot condition the field lines have been pushed to the outer parts of the insulations. This is due to the temperature gradient which creates a gradient in the insulation conductivities.

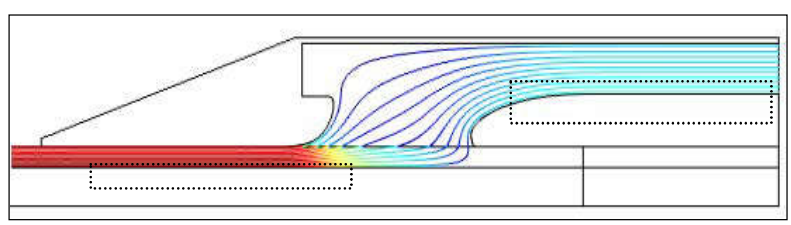

(a)

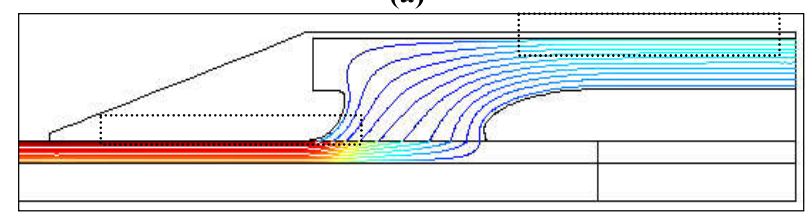

(b)

Fig. 4. Field distribution inside the simulated joint at 278 $\mathrm{kV}$, (a) $20{ }^{\circ} \mathrm{C}$ (b) $70{ }^{\circ} \mathrm{C}$; the dotted boxes indicate the position of the highest local fields in the cable and joint insulation materials. The color scale used is rainbow; from red: high to blue: low field level.

This effect can be observed more clearly in Fig. 5, which shows the normalized tangential electric field across the joint-cable interface at $20{ }^{\circ} \mathrm{C}$ and $70{ }^{\circ} \mathrm{C}$, respectively. In both cases the electric field shows two peaks, at the inner and outer deflectors. However, at room temperature the maximum field is found close to the inner deflector, whereas at high temperature, it is near the outer deflector. This means that during the heat cycling, the tangential field at the interface would change considerably. Due to the temperature distribution in the hot condition, the insulation conductivity increases more, close to the inner deflector than near the outer deflector, which in turn forces the tangential field towards the outer deflector. From Fig. 5, it is clear that the hot condition is more critical for this particular design. This is especially dangerous because the breakdown strength at the interface decreases at higher temperatures [13]. Thus, for an optimized design, the tangential field at high temperatures should be minimized.

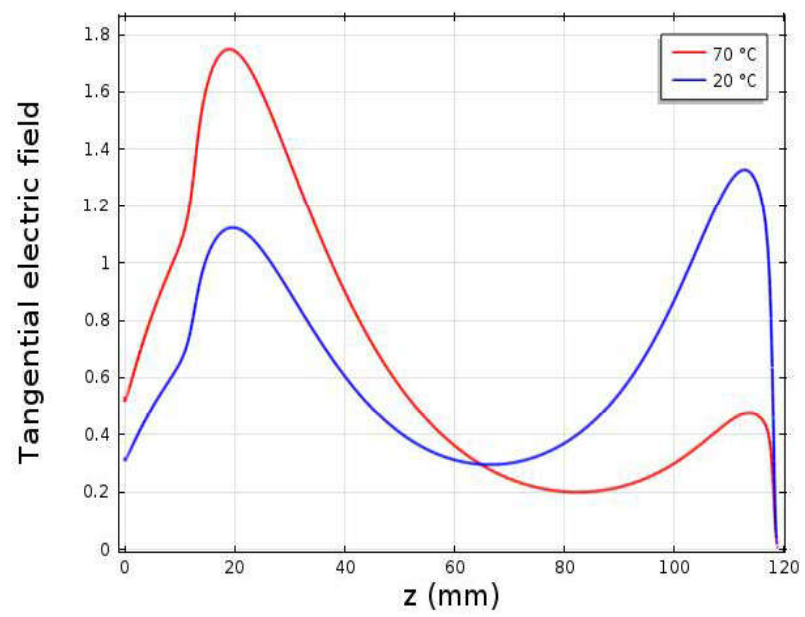

Fig. 5. Normalized tangential electric fields along the cable-joint interface with $278 \mathrm{kV}\left(\mathrm{U}_{\mathrm{T}}\right)$. The outer and inner deflector triple points are located at $0 \mathrm{~mm}$ and 119 $\mathrm{mm}$, respectively.

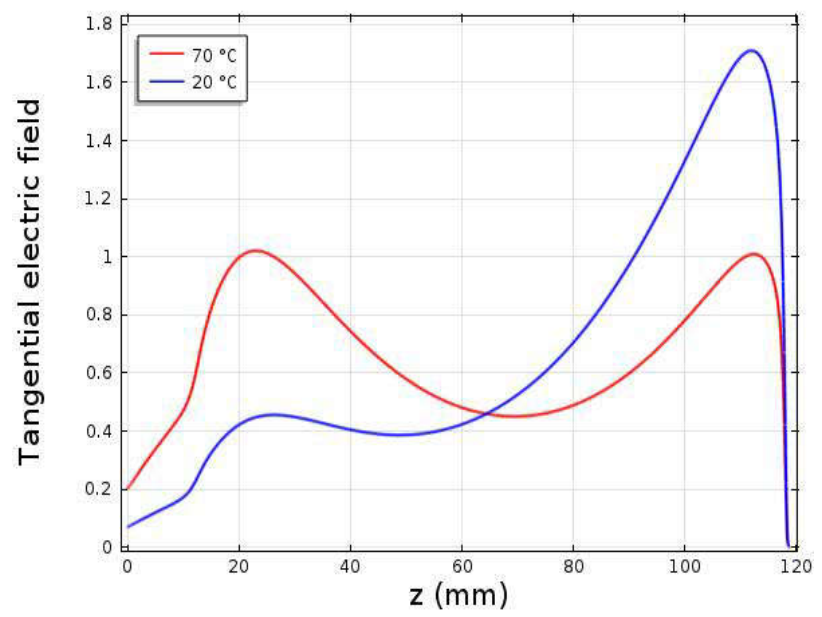

Fig. 6. Normalized tangential electric fields along the cable-joint interface of the Hot Run Design with $278 \mathrm{kV}$ 
One solution to the high tangential field at hot condition could be to modify the joint insulation material. In this design (Hot Run Design), we can achieve this by using EPDM insulation with higher conductivity. By increasing the conductivity a factor 10 it was possible to balance the tangential field at $70{ }^{\circ} \mathrm{C}$. Fig. 6 shows the normalized tangential fields in this case. In comparison to Fig. 5, the tangential field at $70{ }^{\circ} \mathrm{C}$ has a lower maximum field value and the two peaks have the same height. But in this case, the tangential field at room temperature is now extremely unbalanced. Compared to the previous design, the highest field for the cold condition has increased by more than $30 \%$.

Thus, by optimizing the tangential field for high temperatures, the risk at low temperature increases drastically. In addition, large changes occur in the electrical field profile during the temperature cycling tests. Both of these problems are due to the different temperature dependences of XLPE and EPDM conductivities. Moreover, unlike the permittivity, the conductivity of insulation materials varies between different batches and also during their life time.

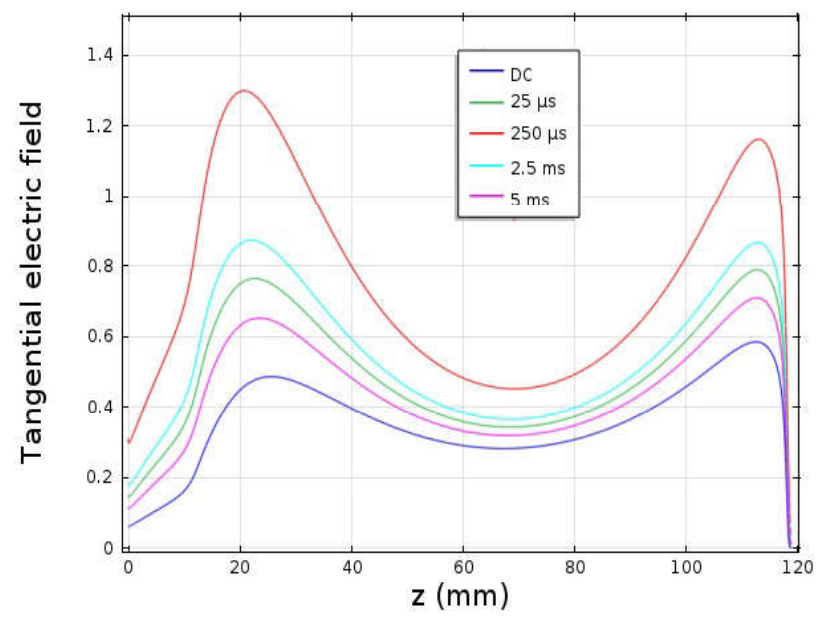

(a)

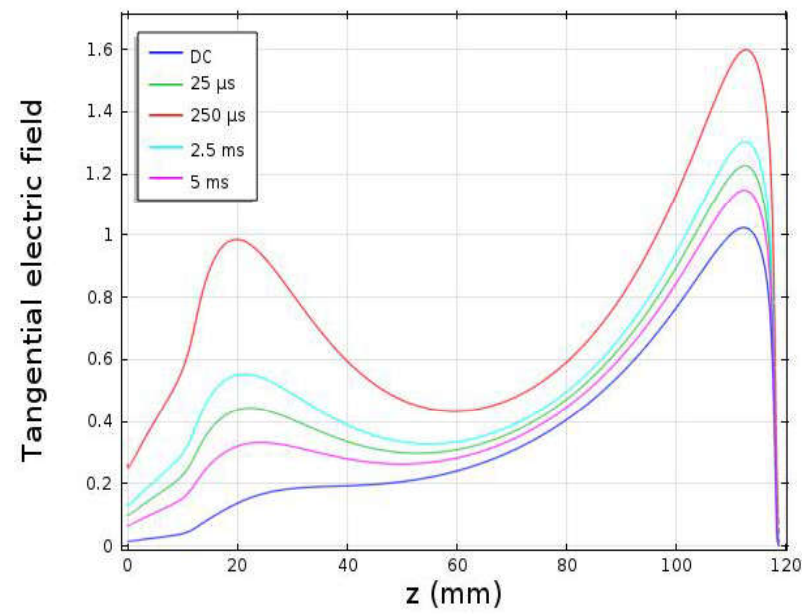

(b)

Fig. 7. Normalized tangential electric fields along the cable-joint interface of the Hot Run Design for a SISP of $315 \mathrm{kV}$ at (a) $70^{\circ} \mathrm{C}$ (b) $20^{\circ} \mathrm{C}$

\subsection{Impulse simulations}

The cable systems should also pass impulse tests. The tangential field at the interface for the Hot Run Design is calculated during the impulse, both at room temperature and at $70{ }^{\circ} \mathrm{C}$. Fig. 7 shows the results from SISP, and Fig. 8 shows the fields during SIOP.

Looking at Fig. 7(a) and (b), the worst case for SISP is the cold condition in which the peak increased $60 \%$ from its already high DC level. Generally, for this design, the SI field stresses the outer deflector more than the inner. The fields would be more critical if the SISP was applied at the hot condition to the initial joint design with DC fields shown in Fig. 5.

If we now focus on the SIOP simulations, according to Fig. 8(b), the peak field is in the same range as the SISP fields in Fig. 7(b) but in the opposite direction. The opposite polarity of the SI in this case would make it more prone to breakdown because of the existing space charges which are accumulated at the interface. Therefore, it is likely that the cold run SIOP is more critical than the cold run SISP.

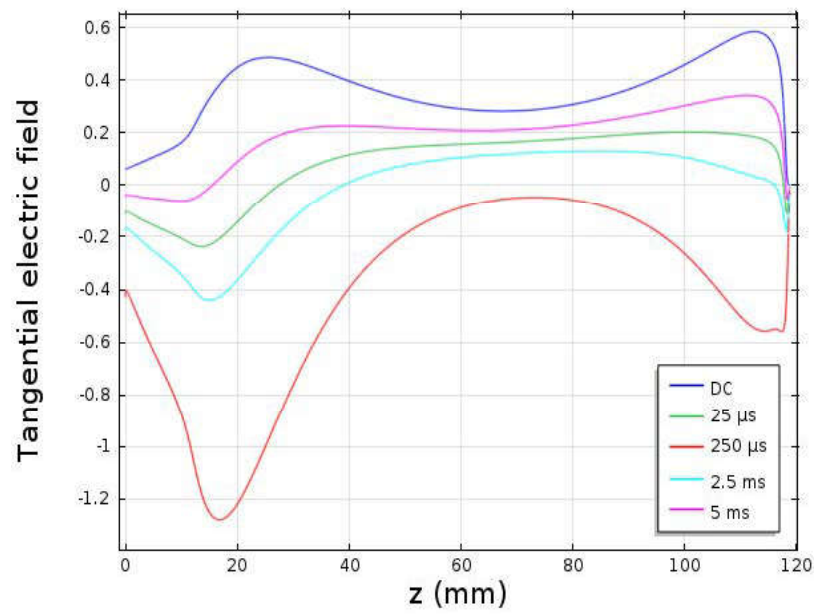

(a)

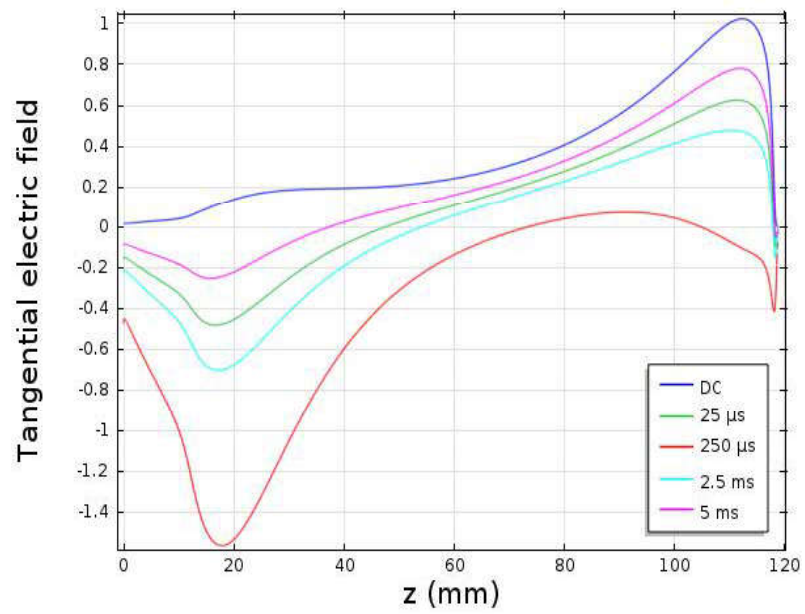

(b)

Fig. 8. Normalized tangential electric fields along the cable-joint interface of the Hot Run Design for a SIOP of $175 \mathrm{kV}$ at (a) $70^{\circ} \mathrm{C}$ (b) $20^{\circ} \mathrm{C}$.

\subsection{Cable conductivity}

The next analysis was to investigate the possibility of increasing the robustness of the joint by improving the 
cable insulation resistivity. Fig. 9 shows the tangential electric fields at the hot condition for three different cable resistivity levels. First, normal XLPE that was used for previous simulations, second, XLPE with 10 times lower conductivity, and third, XLPE with 2 times higher conductivity.

The tangential fields are higher for both high and low XLPE conductivity. This is because optimization of the joint is a balancing act between cable and joint insulation conductivities. The implication is that the joint not only has to be matched to specific test conditions, but also to a specific cable.

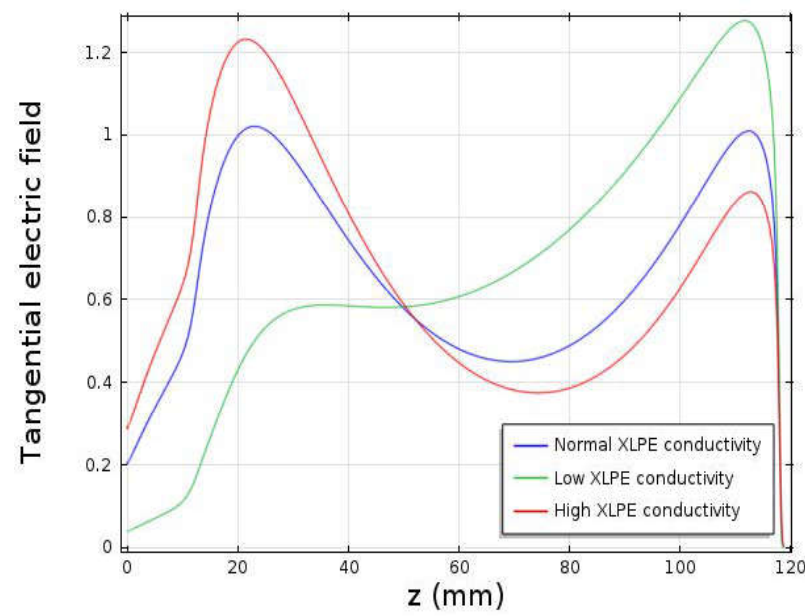

Fig. 9. Normalized tangential electric fields along the cable-joint interface, for the Hot Run Design calculated for different cable XLPE conductivities.

\subsection{Thermal boundary conditions}

A last analysis was performed to study the effect of surface cooling on the joint performance. The level of cooling of a joint depends on the temperature and thermal properties of the soil, or sea, where it is installed, and the joint must be robust enough for a wide range of surrounding conditions. In this study, with the same current in the conductor, the cooling factor of the cable system was varied from good to poor, and the results from this analysis are shown in the Fig. 10.

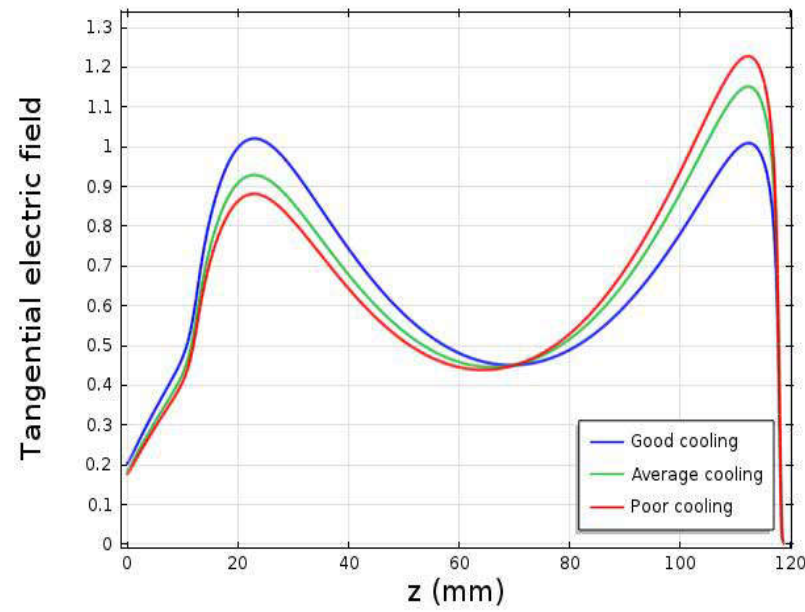

Fig. 10. Normalized tangential electric fields along the cable-joint interface, for Hot Run Design calculated at different surface cooling conditions.
In this study, good cooling roughly approximates convective cooling of the cable and joint in air, whereas the other two represent more realistic soil conditions. In the previous sections, good cooling conditions have been used for all simulations. The results show that the tangential field at the interface is also sensitive to the surface cooling, increasing by $23 \%$ from good to poor cooling conditions. This poses more complications which have to be taken into account during the design and testing of a joint.

\subsection{Solution: Non-linear Field Grading Material}

As mentioned above, the variation of conductivity in cable and joint insulation due to production variations, operation condition and temperature cycling, makes the design of a robust joint very challenging. A good solution to this problem is application of a properly designed FGM between the joint and cable insulations, see Fig. 11. The conductivity of the FGM increases strongly with field and temperature, which will decouple the two insulation materials and therefore the system becomes less sensitive to the conductivity variations of the insulation layers.

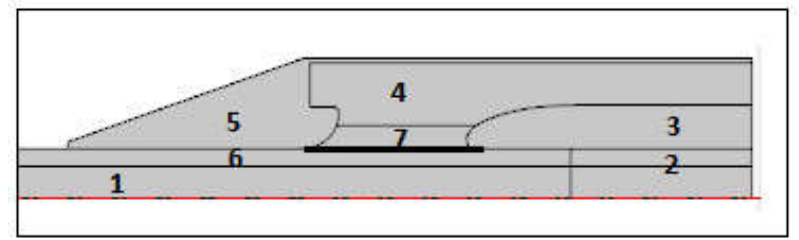

Fig. 11. Generic joint design with FGM used in the simulations. The numbers indicate different parts of the joint: 1-conductor, 2-connector, 3-inner deflector, 4-joint insulation, 5-outer deflector, 6-cable insulation, 7-field grading layer. The thick line indicates the interface between joint and cable insulation materials.

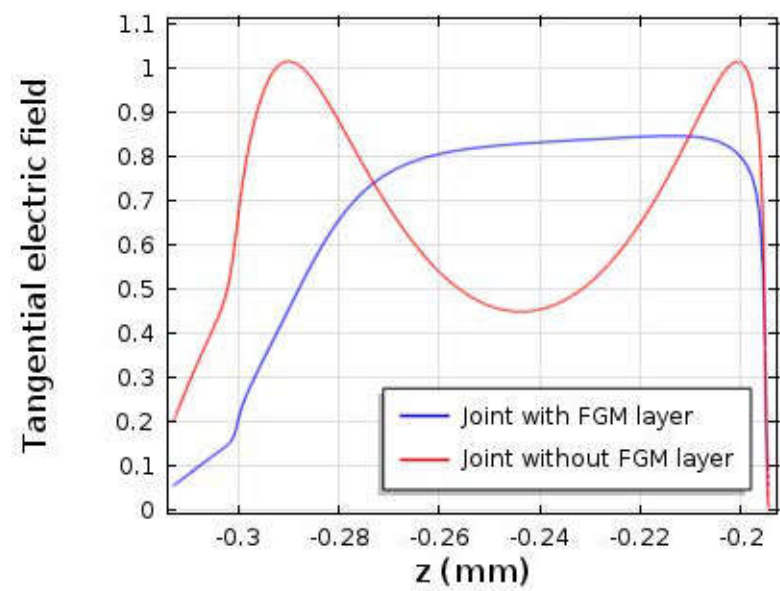

Fig. 12. Normalized tangential electric fields along the cable-joint interface for joints with FGM and without FGM (Hot Run Design), respectively; DC TT voltage in hot condition.

Fig. 12 presents a comparison between the interface fields in the joint with and without FGM layer (Hot Run Design) for DC TT voltage in hot condition. Although no special optimization was done for the design with 
FGM, it has an almost flat tangential field distribution along the interface with a peak value less than that of the optimized Hot Run Design. Since the field distribution at the interface is governed by the FGM conductivity, the field profile is robust to temperature variations and variations in cable and joint insulation materials as shown in [7] in more detail. It is notable that adding a FGM layer, adds two new triple points to the system and one has to design the joint geometry considering these triple points.

\section{Conclusions}

A classical joint design was created and the electric field for different temperature settings and voltages was calculated in COMSOL Multiphysics.

The tangential electric field at the interface between the joint and cable insulations is the most critical parameter to be considered. The field profile varies considerably due to the temperature-dependent conductivities of the cable and joint insulation materials. One needs to carefully tune the conductivity of the joint insulation to get a reasonable match for a certain cable at a certain temperature. Doing so, one can achieve a design which works satisfactory at this specific temperature, e.g. high temperature, but the design will be more prone to failure in other conditions, e.g. low temperatures. Due to the need for a good match between cable and insulation conductivities; first, a joint designed for a certain cable may not work for a different cable. Second, the design will be very sensitive to variations in the produced material batches. Therefore very careful quality control is needed. Finally, the variation of insulation conductivities during their life time may ruin the match which was aimed for in the design.

In Switching Impulse tests the most critical case for a joint designed for DC in hot condition, is the SIOP in cold state. In this case the interface close to the outer deflector would be stressed with high fields in opposite direction to the DC field and may create failure by fast transport of space charges at the interface.

Studying the tangential fields of a joint with tuned insulation conductivity with different surface cooling levels shows that the worst case occurs at the joint with the poorest cooling.

Considering the points presented above, the robustness of designs with this approach is considered as a major concern. This finding encourages the more recent approach to HVDC joint design which is based on nonlinear field grading materials.

\section{References}

[1] Marc Jeroense, Anders Gustafsson, Peter Sunnegård, and L. Carlsson, "The status and future of HVDC Light cable systems," Cigré SC B4, Bergen Colloquium, 2009.

[2] M. Byggeth, Kenneth Johansson, C. Liljegren, and U. Axelsson, "Gotland HVDC Light - The World's first commercial extruded HVDC cable system.," Cigré, 2000, vol. 14, no. 14-205, p. 205.
[3] K Bergman, A Gustafsson, MJP Jeroense, and F Mekic, "Extruded HVDC cables - a solution for different customer segments," Cigré colloquium on HVDC and power electronic system for overhead line and insulated cable applications, San Francisco, March 7-9, 2012, B1-7.

[4] Thomas Christen, Lise Donzel, and Felix Greuter, "Nonlinear resistive electric field grading part 1: theory and simulation," IEEE Electrical Insulation Magazine, 2010, vol. 26, no. 6, pp. 47-59.

[5] Thomas Christen, Lise Donzel, Felix Greuter, and Markus Saltzer, "Fundamentals of resistive field grading," Feldsteuernde Isoliersysteme, 2011, vol. ETG-FB 131.

[6] Lise Donzel, Felix Greuter, and Thomas Christen, "Nonlinear resistive electric field grading part 2: materials and applications," IEEE Electrical Insulation Magazine, 2011, vol. 27, no. 2, pp. 1829.

[7] Markus Saltzer, Thomas Christen, Torbjörn Sörqvist, and Marc Jeroense, "Electro-thermal simulations of HVDC cable joints," Feldsteuernde Isoliersysteme, 2011, vol. ETG-FB 131.

[8] Hampton, R.N.; Chang, F.; Hobdell, S.: What happens to materials under HVDC?, Cigre 2000 Session papers , P2-001, 2000.

[9] Cigré and , "Recommendations for tests of power transmission DC cables for a rated voltage up to 600 kV," Electra, 1980, vol. 72, no. 72, pp. 105 114.

[10] Chengwei Chen, Gang Liu, Guojun Lu, and Jin Wang, "Mechanism on breakdown phenomenon of cable joint with impurities," Proceedings of the 9th International Conference on Properties and Applications of Dielectric Materials, Harbin, China, July 19-23, 2009, pp. 595-597.

[11] J.A. Pilgrim, D.J. Swaffield, P.L. Lewin, and D. Payne, "An investigation of thermal ratings for high voltage cable joints through the use of $2 \mathrm{D}$ and 3D finite element analysis," Conference proceedings of the 2008 IEEE International Symposium on Electrical Insulation, 9-12 June, 2008, pp. 543-546.

[12] D. Fournier and L. Lamarre, "Effect of pressure and length on interfacial breakdown between two dielectric surfaces," Conference record of the 1994 IEEE International Symposium on Electrical Insulation, Baltimore, MD, USA, June 7-10, 1992, pp. 270-272.

[13] Normand Amyot and Daniel Fournier, "Influence of thermal cycling on the cable-joint interfacial pressure," 2001 IEEE 7th International Conference on Solid Dielectrics, 2001, pp. 35-38.

[14] Marc Jeroense et al., "Increased voltage for the HVDC Light $\AA$ product range - a complete solution," JICABLE07, vol. B47, 2007. 